\title{
Adiabatic index of hot and cold compact objects
}

\author{
R. H. Casali and D. P. Menezes \\ Departamento de Física - CFM - Universidade Federal de Santa Catarina \\ - Florianópolis - SC - Caixa Postal 476 - CEP 88.040-900 - Brazil
}

(Received on 16 September, 2009)

\begin{abstract}
In the present work we calculate the adiabatic index of neutron stars formed by hadronic or quarkionic matter, under two possible conditions, with and without trapped neutrinos, and for two values of the entropy per baryon $S=0,2 k_{B}$ / baryon. We use the nonlinear Walecka model to describe hadronic stars, and the MIT bag model to describe quark stars. Particle fractions for each case are obtained and the appearance of particles are compared with drops in the adiabatic index, whenever possible.
\end{abstract}

Keywords: Neutron stars; Quark stars; Adiabatic index

\section{INTRODUCTION}

The adiabatic index $(\Gamma)$ is an important thermodynamical quantity. In a classical theory it is related to both the specific heat at constant pressure and the specific heat at constant volume $\left(\Gamma=C_{p} / C_{v}\right)$.

Compact stars are objects whose constituents can be used to build their equations of state (EOS) and consequently their adiabatic index, used to infer their stability [1,2]. The composition of neutron stars remains a source of speculation. Three of the possibilities are that 1) the stars are formed by hadrons plus leptons (hadronic stars) [3], 2) they are formed by quarks plus leptons (strange or quark stars) [4] and 3) that they are hybrid, formed by hadrons plus leptons and quarks plus leptons, bearing [5] or nor a mixed phase [6].

Polytropes are equations of state for which the adiabatic index is constant. A free Fermi non-relativistic (relativistic) gas can be described by an EOS with $\Gamma=5 / 3(4 / 3)$. If the fermions are electrons, the resulting polytropic EOS can be used to describe white dwarfs. If they are neutrons, they describe neutron stars $[7,8]$.

In $[9,10]$ piecewise polytropic EOSs are used to describe neutron star properties. In [10], a 4-parameter polytrope is used to fit hybrid star EOS obtained with more fundamental models, where the crust has an index $\Gamma_{1}$, the hadronic phase $\Gamma_{2}$, the mixed phase an index $\Gamma_{3}$ and the core constituted by quarks an index $\Gamma_{4}$. This piecewise EOS is then used in a study of constraints placed by astrophysical observations on neutron stars.

In the present work we calculate the adiabatic index for hadronic and quark stars both for zero temperature and fixed entropy per baryon. The non-linear Walecka model [11] is used to obtain the EOS for hadronic matter and the MIT bag model [12] for quark matter.

The paper is organized as follows: in Section II the basic formalism used to describe hadronic and quark matter is briefly described. In Section III the results are presented and discussed and in the Section IV the final conclusions are drawn.

\section{THE FORMALISM}

We next describe the basic equations for the relativistic models used in the present work.

\subsection{Hadronic stars}

The Lagrangian density for the non-linear Walecka model can be written as [11]:

$$
\mathcal{L}_{H}=\mathcal{L}_{N L W M}+\mathcal{L}_{l},
$$

where

$$
\begin{aligned}
\mathcal{L}_{N L W M}= & \sum_{B} \bar{\psi}_{B}\left[\gamma_{\mu}\left(l \partial^{\mu}-g_{\nu B} V^{\mu}-g_{\rho B} \vec{\tau} \cdot \mathbf{b}^{\mu}\right)\right. \\
& \left.-M_{B}^{*}\right] \psi_{B}+\frac{1}{2}\left(\partial_{\mu} \phi \partial^{\mu} \phi-m_{s}^{2} \phi^{2}\right)-\frac{1}{3 !} k \phi^{3} \\
& -\frac{1}{4 !} \lambda \phi^{4}-\frac{1}{4} \Omega_{\mu v} \Omega^{\mu \nu}+\frac{1}{2} m_{v}^{2} V_{\mu} V^{\mu} \\
& -\frac{1}{4} \mathbf{B}_{\mu \nu} \cdot \mathbf{B}^{\mu \nu}+\frac{1}{2} m_{\rho}^{2} \mathbf{b}_{\mu} \cdot \mathbf{b}^{\mu}
\end{aligned}
$$

and

$$
\mathcal{L}_{l}=\sum_{l=e^{-}, \mu^{-}} \bar{\psi}_{l}\left(l \gamma_{\mu} \partial^{\mu}-m_{l}\right) \psi_{l}
$$

with $\Sigma_{B}$ extending over the eight baryons and where $g_{i B}$ and $m_{i}$ are respsctively the coupling constants of the mesons $i=$ $s, v, \rho, \delta$ with the hyperons and their masses. Self-interacting terms for the $\sigma$-meson are also included, $k$ and $\lambda$ denoting the corresponding coupling constants and $\tau$ is the isospin operator. The set of coupling constants of the baryons of the octect and the mesos are $g_{s B}=x_{s B} g_{s}, g_{v B}=x_{v B} g_{v}, g_{\rho B}=x_{\rho B} g_{\rho}$, and $x_{s B}, x_{v B}$ and $x_{\rho B}$ are equal to 1 for the nucleons. We have chosen $x_{S B}=0.7$ and $x_{v B}=x_{\rho B}=0.783$ and assumed that the couplings to the $\Sigma$ and $\Xi$ are equal to those of the $\Lambda$ hyperon[11].As parameters we have used [11] $g_{s}^{2} / m_{s}^{2}=$ $11.79 \mathrm{fm}^{2}, g_{v}^{2} / m_{v}^{2}=7.148 \mathrm{fm}^{2}, g_{\rho}^{2} / \mathrm{m}_{\rho}^{2}=4.410 \mathrm{fm}^{2}, \mathrm{k} / \mathrm{M}=$ 4.1684, $\lambda=-41.3685$, for which the binding energy is $-16.3 \mathrm{MeV}$ at the saturation density $\rho_{0}=0.153 \mathrm{fm}^{-1}$, the symmetry coefficient is $32.5 \mathrm{MeV}$ and the compression modulus is $300 \mathrm{MeV}$ [11]. $M_{B}^{*}=\left(M_{B}-g_{s B} \phi\right)=0.70 \mathrm{M}$ at the saturation density represents the effective mass of each baryon. This parametrizations is often called GM1 in the literature.

After the application of the Euler-Lagrange equations to the Lagrangian density, the equations of motion for the nucleons and mesons read

$$
\phi_{0}=-\frac{k}{2 m_{s}^{2}} \phi_{0}^{2}-\frac{\lambda}{6 m_{s}^{2}} \phi_{0}^{3}+\sum_{B} \frac{g_{s}}{m_{s}^{2}} x_{s B} \rho_{s B},
$$




$$
\begin{gathered}
V_{0}=\sum_{B} \frac{g_{v}}{m_{v}^{2}} x_{v B} \rho_{B}, \\
b_{0}=\sum_{B} \frac{g_{\rho}}{m_{\rho}^{2}} x_{\rho B} \tau_{3 B} \rho_{B},
\end{gathered}
$$

where:

$$
\rho_{s B}=\frac{1}{2 \pi} \int p^{2} d p \frac{M_{B}^{*}}{\varepsilon}\left[f_{B+}+f_{B-}\right]
$$

and

$$
\rho_{B}=\frac{1}{2 \pi} \int p^{2} d p\left[f_{B+}-f_{B-}\right]
$$

The distribution functions for baryons and anti-baryons are:

$$
f_{B \pm}=\frac{1}{1+e^{\left[\left(\varepsilon_{B} \mp v_{B 0}\right) / T\right]}}
$$

with

$$
\varepsilon_{B}=\sqrt{p^{2}+M_{B}^{*}}, \quad v_{B 0}=\mu_{B}-g_{\vee B} V_{0}-g_{\rho B} \tau_{3 B} b_{0}
$$

The expressions for the energy density $\varepsilon$ and pressure $\mathcal{P}$ for the hadronic model are given by [11]:

$$
\varepsilon=\varepsilon_{N L W M}+\varepsilon_{l}
$$

with:

$$
\begin{aligned}
& \varepsilon_{N L W M}=\frac{1}{\pi^{2}} \sum_{B} \int p^{2} d p \sqrt{p^{2}+M_{B}^{* 2}}\left(f_{B+}+f_{B-}\right) \\
& +\frac{m_{v}^{2}}{2} V_{0}^{2}+\frac{m_{\rho}^{2}}{2} b_{0}^{2}+\frac{m_{s}^{2}}{2} \phi_{0}^{2}+\frac{k}{6} \phi_{0}^{3}+\frac{\lambda}{24} \phi_{0}^{4},
\end{aligned}
$$

the energy density of the leptons is:

$$
\varepsilon_{l}=\frac{1}{\pi^{2}} \sum_{l} \int p^{2} d p \sqrt{p^{2}+m_{l}^{2}}\left(f_{l-}+f_{l+}\right),
$$

and

$$
\mathcal{P}=\mathcal{P}_{N L W M}+\mathcal{P}_{l}
$$

with

$$
\begin{aligned}
& \mathcal{P}_{N L W M}=\frac{1}{3 \pi^{2}} \sum_{B} \int \frac{p^{4} d p}{\sqrt{p^{2}+M^{* 2}}}\left(f_{B+}+f_{B-}\right) \\
& +\frac{m_{v}^{2}}{2} V_{0}^{2}+\frac{m_{\rho}^{2}}{2} b_{0}^{2}-\frac{m_{s}^{2}}{2} \phi_{0}^{2}-\frac{k}{6} \phi_{0}^{3}-\frac{\lambda}{24} \phi_{0}^{4},
\end{aligned}
$$

the pressure of the leptons is:

$$
\mathcal{P}_{l}=\frac{1}{3 \pi^{2}} \sum_{l} \int \frac{p^{4} d p}{\sqrt{p^{2}+m_{l}^{2}}}\left(f_{l-}+f_{l+}\right),
$$

with the lepton distribution functions given by:

$$
f_{l \pm}=\frac{1}{1+e^{\left[\left(\varepsilon_{l} \mp \mu_{l}\right) / T\right]}},
$$

where $\mu_{l}$ is the chemical potential of the leptons and:

$$
\varepsilon_{l}=\sqrt{p_{l}^{2}+m_{l}^{2}}
$$

From the thermodynamic potential the entropy density of the system can be written as:

$$
s=\frac{1}{T}\left(\mathcal{P}+\varepsilon-\sum_{B} \mu_{B} \rho_{B}-\sum_{l} \mu_{l} \rho_{l}\right),
$$

where the density of the leptons is:

$$
\rho_{l}=2 \int \frac{d^{3} p}{\left(2 \pi^{3}\right)}\left(f_{l+}-f_{l-}\right) .
$$

The condition of chemical equilibrium implies that:

$$
\mu_{n}=\mu_{p}+\mu_{e^{-}}+\mu_{\bar{v}_{e^{-}}} .
$$

The neutrino term in the above equation refers to an initial stage of the neutron star, when the neutrinos are still trapped in its interior. After this stage the chemical equilibrium condition becomes:

$$
\mu_{n}=\mu_{p}+\mu_{e^{-}}
$$

$$
\mu_{\Sigma^{0}}=\mu_{\Xi^{0}}=\mu_{\Lambda}=\mu_{n},
$$

$$
\begin{gathered}
\mu_{\Sigma^{-}}=\mu_{\Xi^{-}}=\mu_{n}+\mu_{e^{-}}, \\
\mu_{\Sigma^{+}}=\mu_{p}=\mu_{n}-\mu_{e^{-}}, \\
\mu_{\mu^{-}}=\mu_{e^{-}} .
\end{gathered}
$$

The charge neutrality implies that:

$$
\sum_{B} q_{B} \rho_{B}=\rho_{e^{-}}+\rho_{\mu^{-}}
$$

\subsection{Quark stars}

The MIT bag model [12] is used to describe quark matter. In this model the quarks are considered to be free in a bag. The energy density for the bag model can be written as:

$$
\varepsilon=\varepsilon_{M I T}+\varepsilon_{l}
$$

with

$$
\begin{aligned}
& \varepsilon_{M I T}=3 \times 2 \sum_{q=u, d, s} \int \frac{d^{3} p}{(2 \pi)^{3}} \sqrt{p^{2}+m_{q}^{2}}\left(f_{q+}+f_{q-}\right) \\
& +B,
\end{aligned}
$$


where the value 3 represents the number of colors, 2 represents the spin degeneracy, $m_{q}$ is the mass of each quark and the term $B$ represents the bag pressure [12]. The expression for $\varepsilon_{l}$ is given by the equation (12) and the pressure can be written as:

$$
\mathcal{P}=\mathcal{P}_{M I T}+\mathcal{P}_{l}
$$

with

$$
\begin{aligned}
& \mathcal{P}_{M I T}=\frac{1}{\pi^{2}} \sum_{q=u, d, s} \int \frac{d p p^{4}}{\sqrt{p^{2}+m_{q}^{2}}}\left(f_{q+}+f_{q-}\right) \\
& -B,
\end{aligned}
$$

and the term $\mathcal{P}_{l}$ is given by the equation (15). The density of the quarks is given by:

$$
\rho_{q}=\frac{2 \times 3}{2 \pi^{2}} \int p^{2} d p\left[f_{q+}-f_{q-}\right],
$$

and the distribution function for the quarks and anti-quarks are given by the Fermi distribution:

$$
f_{q \pm}=\frac{1}{\left(1+e^{\left[\left(\varepsilon \mp \mu_{q}\right) / T\right]}\right)},
$$

with $\varepsilon=\sqrt{p^{2}+m_{q}^{2}}, \mu_{q}$ or $\left(-\mu_{q}\right)$, being the values of the chemical potentials of the quarks or (anti-quarks) of type $q$. We have used $m_{u}=m_{d}=5.5 \mathrm{MeV}, m_{s}=150 \mathrm{MeV}$, $B=(150 \mathrm{MeV})^{4}$.

The relations between the chemical potential of the different particles imposed by chemical equilibrium are:

$$
\begin{gathered}
\mu_{s}=\mu_{d}=\mu_{u^{-}}+\mu_{e^{-}}, \\
\mu_{e^{-}}=\mu_{\mu^{-}} .
\end{gathered}
$$

For the charge neutrality we have imposed:

$$
\rho_{e}+\rho_{\mu}=\frac{1}{3}\left(2 \rho_{u}-\rho_{d}-\rho_{s}\right) \text {. }
$$

\subsection{Adiabatic Index}

For stars, the stability is related with the value of the adiabatic index in its interior that has to be larger than $\frac{4}{3}$ [13]. Our goal in the present work is to study the behavior of this parameter of the matter that constitutes the star. We use the expression [14]:

$$
\Gamma=\frac{\varepsilon}{P} \frac{d P}{d \varepsilon},
$$

where $P$ and $\varepsilon$ stand respectively for the pressure and energy density of the model under consideration.

\section{RESULTS}

In the following we discuss the results obtained for hadronic and quark stars. Not only the adiabatic index, but also the corresponding particle fractions are displayed for each case. Whenever possible a correlation between the appearence of the particles and the maxima and minima in the adiabatic index is established.

\subsubsection{Hadronic stars}

Figs. 1 and 2 show the particle fractions versus density for entropies $S=0 k_{B}$ /baryon (upper panel) and $S=2 k_{B} /$ baryon (lower panel), for the nonlinear Walecka model.

Fig. 1 shows the fractions of hyperons, nucleons, and leptons in a system with trapped neutrinos and fixed lepton fraction $Y_{l}=0.4$. In the upper panel all species appear up to $\rho \sim 0.8 \mathrm{fm}^{-3}$. For $S=2 k_{B}$ /baryon the baryons $\Xi^{-}$and $\Xi^{0}$ already emerge at $\rho \sim 0.4 \mathrm{fm}^{-3}$. At this point it is important to emphasize that the case $S=0$ and trapped neutrinos is displayed for academic purpose and for the sake of comparison with the other cases. It is well known that at zero temperature no trapped neutrinos are left in the star because their mean free path would be larger than the compact star radius.
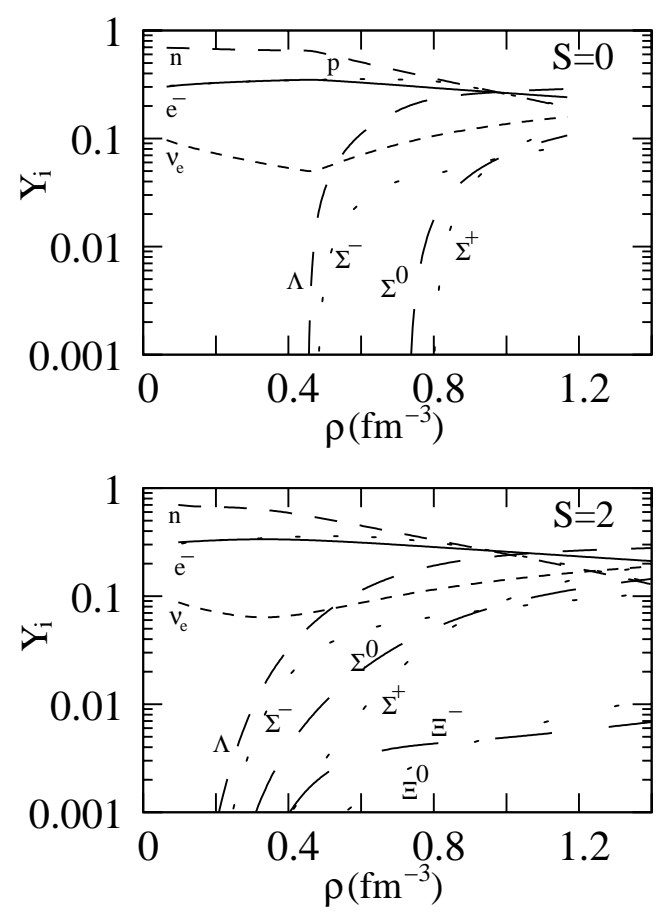

FIG. 1: Particle fractions for a hadronic star whit trapped neutrinos.

In Fig. 2 the particle fractions in a system without trapped neutrinos are shown. In the upper panel, even after $\rho \sim$ $0.8 \mathrm{fm}^{-3}$ baryonic species are emerging. In the lower panel, one can see that all species emerge before $\rho \sim 0.5 \mathrm{fm}^{-3}$ and the baryonic distribution stabilizes after $\rho \sim 1.0 \mathrm{fm}^{-3}$. In both cases we can see that the electric charge neutrality is conserved, while positively charged particles appear, the negative hyperons have their population increased.

As expected the heaviest hyperons emerge at higher densities. These species populate the inner regions of neutron stars. As entropy increases, hyperons turn up at lower densities. 

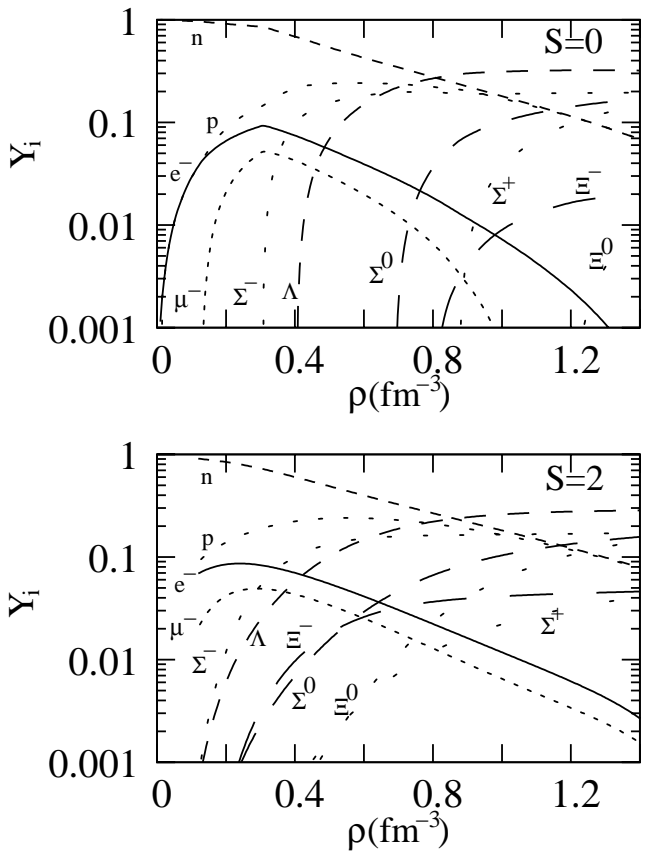

FIG. 2: Particle fractions for a hadronic star whitout trapped neutrinos.

Fig. 3, shows the behavior of the adiabatic index $\Gamma$ versus the baryonic density $\rho$ for $S=0 k_{B}$ /baryon (upper panel) and for $S=2 k_{B}$ /baryon (lower panel) for stars with and without trapped neutrinos. For $S=0 k_{B}$ /baryon, we can identify the apperance of particles with deflections in the curves of the adiabatic index. For $S=2 k_{B}$ /baryon the identification is not possible.
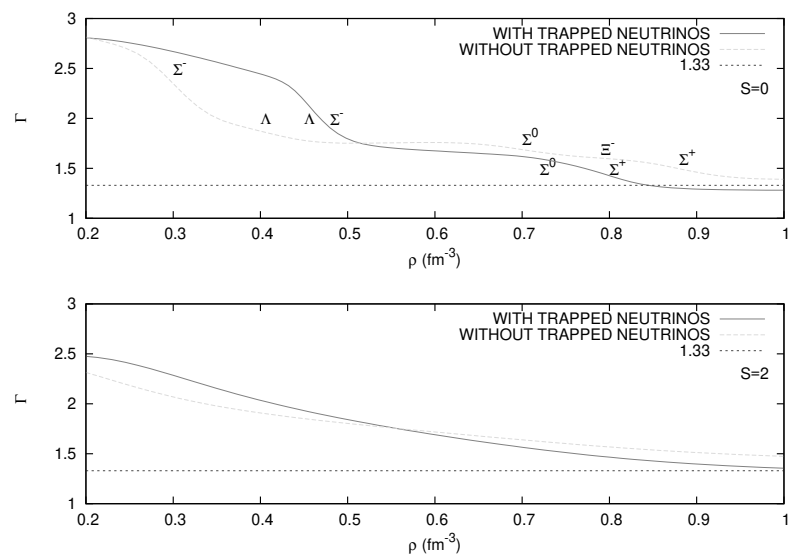

FIG. 3: The adiabatic index $\Gamma$ versus density $\rho$.

The horizontal line sets the threshold of stability. As indicated in the upper pannel, the adiabatic index drops at densities that correspond to specific hyperon thresholds. After the thresholds the equation of state is softened because of the new hyperon. The case with trapped neutrinos presents a region ( $\rho \sim 0.85 \mathrm{fm}^{-3}$ ) where the adiabatic index becomes lower than the stability threshold $\frac{4}{3}$. Notice that this only happens in the case referred to the non physical situation of zero temperature and trapped neutrinos.
In the lower pannel with higher entropy the behavior of the adiabatic index seems to be always stable no matter the value of the density.

\subsubsection{Quarkionic stars}

Figs. 4 and 5 show particle fractions for entropies $S=$ $0 k_{B}$ /baryon (upper panel) and $S=2 k_{B}$ /baryon (lower panel), for the MIT bag model.

In Fig. 4 we display a system consisting of quarks $u, d, s$ and leptons $e^{-}$and $v_{e}$. For both cases the particle fractions are practically the same. The neutrino content increases at lower densities and then reaches a maximum value and stabilizes.

The electron fraction has a little decrease till it stabilizes, and its presence restricts the s quark content due to the electric charge conservation.

Fig. 5 shows the $u, d, s$ quarks and $e^{-}$fractions, without trapped neutrinos. For the two entropies considered the electron content decreases quickly.

In Fig. 6 we plot the adiabatic index $\Gamma$ versus density $\rho$ for $S=0 k_{B}$ /baryon (upper panel) and for $S=2 k_{B}$ /baryon (lower panel) and the limit of stability. For both values of entropy, $\Gamma$ shows practically the same behavior for stars with and without trapped neutrinos, decaying slowly but never crossing the threshold of stability.
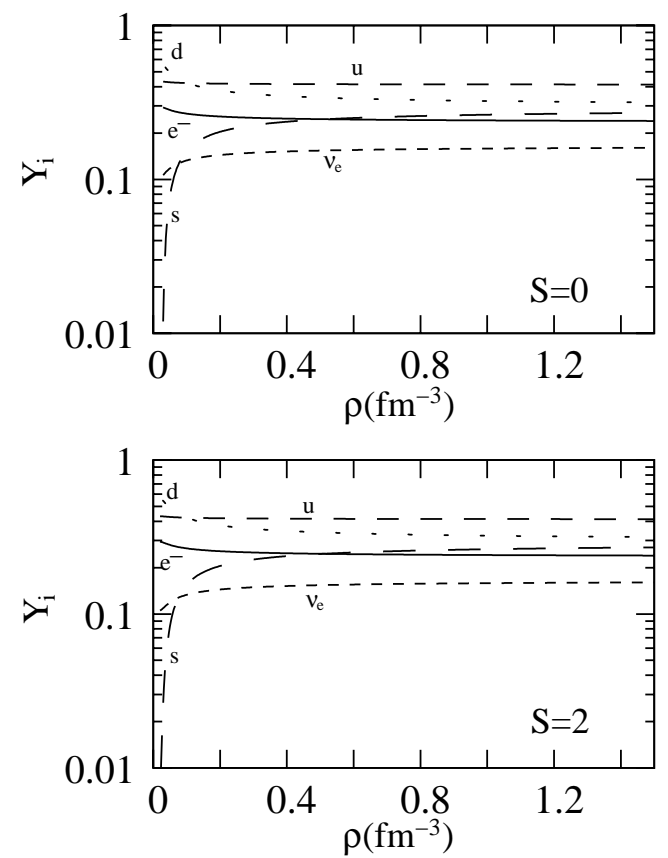

FIG. 4: Particle fractions for a quarkionic star whit trapped neutrinos.

\section{CONCLUSIONS}

In the present work we have studied the adiabatic index of neutron stars with and without trapped neutrinos formed by hadronic or quarkionic matter for two values of the entropy 

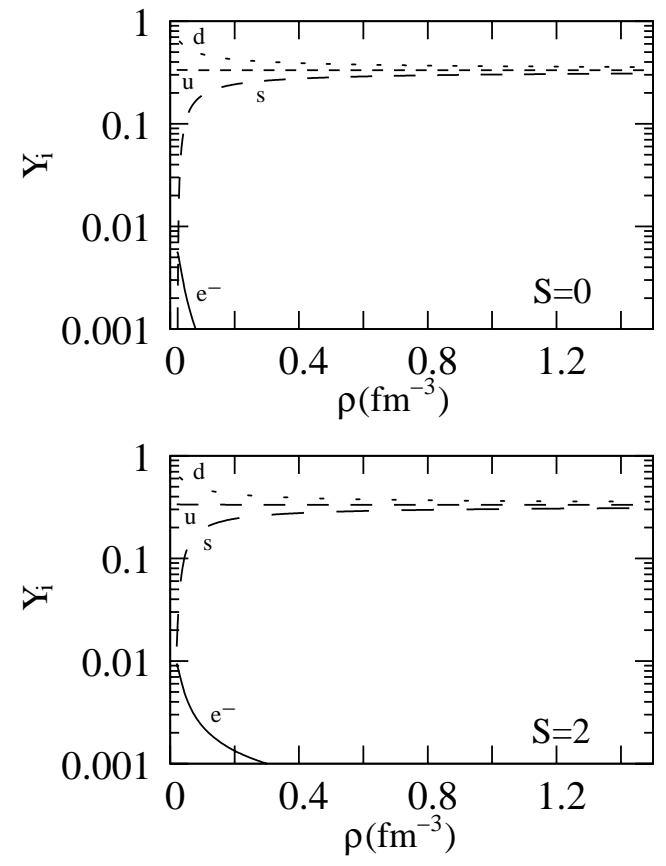

FIG. 5: Particle fractions for a quarkionic star whitout trapped neutrinos.
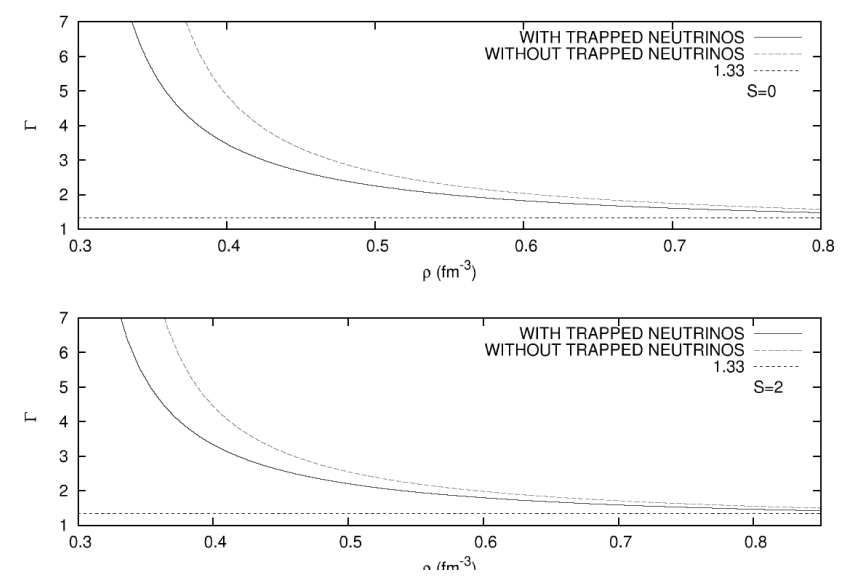

FIG. 6: The adiabatic index $\Gamma$ versus density $\rho$, for
$S=0,2 k_{B}$ /baryon. We have used the NLWM and the MIT bag model to describe these systems.

We have concluded that for hadronic stars and zero temperature $(S=0)$, there is a clear correlation between the appearance of particles and the behaviour of the adiabatic index. For larger entropies and for quark stars the correlation does not exist or is not obvious.

An obvious continuation of the present work would be the calculation of the adiabatic index for various values of the entropy between zero and 2 in order to mimic a protoneutron star evolution. The results could help the understanding of its stability during the deleptonization and cooling processes.

Once the adiabatic index for hadronic and quarkionic stars was obtained, the same procedure can be repeated in the investigation of $\Gamma$ for hybrid stars. Contrary to what was done with piecewise polytropic EOS in [10], a continuous EOS as obtained in [5] can be used for the calculation of the adiabatic index. This work is under investigation.

\section{Acknowledgments}

This work was partially supported by CNPq (Brazil).

[1] R.B. Jacobsen, Plasma de quarks e gluons no interior de estrelas de nêutrons, Msc. dissertation, Instituto de Fsica, Universidade Federal do Rio Grande do Sul, 2007.

[2] R.H. Casali,Objetos estelares compactos quentes e seus índices adiabáticos, Msc. dissertation, Programa de Pós-Graduação em Física, Universidade Federal de Santa Catarina, 2008.

[3] A. L. Espíndola and D. P. Menezes, Phys. Rev. C 65, 045803 (2002); A.M.S. Santos and D.P. Menezes, Phys. Rev. C 69, 045803 (2004); R. Cavagnoli and D.P. Menezes, Braz. J. Phys. 35 B, 869 (2005); Jour. Phys. G 35, 115202 (2008).

[4] D.P. Menezes, C. Providência, D.B. Melrose, J.Phys. G 32,1081 (2006).

[5] D.P. Menezes and C. Providência, Phys. Rev. C 68, 035804 (2003); D.P. Menezes and C. Providência, Phys. Rev.C 69,
045801 (2004); P.K. Panda, D.P. Menezes and C. Providência, Phys. Rev. C 69, 025207 (2004); P.K. Panda, D.P. Menezes and C. Providência, Phys. Rev. C 69, 058801 (2004).

[6] T. Tatsumi, M. Yasuhira and D.N. Voskresensky, Nucl. Phys. A 718 (2003) 359c; Yu. B. Ivanov, A.S. Khvorostukhin, E.E. Kolomeitsev, V.V. Skokov, V.D. Toneev and D.N. Voskresensky, Phys. Rev. C 72, 025804 (2005).

[7] R. Tooper, Astrophys. J., 140, 434 (1964).

[8] L. Ferrari, G. Estrela and M. Malheiro, Inter. J. of Mod. Phys. E 16, 2834 (2007).

[9] J. L. Zdunik, M. Bejger, P. Haensel and E. Gourgoulhon, A \& A 450, 747 (2006); M. Bejger, P. Haensel and J. L. Zdunik, MNRAS 359, 699 (2005); P. Haensel and A. Y. Potekhin, A \& A 428, 191 (2004). 
[10] J.S. Read,B.D. Lackey, B.J. Owenand J.L. Friedam, arXiv:0812.2163v1 [astro-ph].

[11] Glendenning N. K.,Compact Stars. Springer-Verlag: NewYork, (2000).

[12] A. Chodos, R.L. Jaffe, K. Johnson, C.B. Thorne and V.F. Weisskopf, Phys. Rev. D 9, 3471 (1974).
[13] M. Onsi, H. Przysiezniak, J.M. Pearson, Phys. Rev. C 50, 1 (1994).

[14] L.Ferrari, P.C.R.Rossi and M. Malheiro, to be published in Int. J. of. Mod. Phys. D. 